\title{
Customer Social Network Affects Marketing Strategy: A Simulation Analysis Based on Competitive Diffusion Model
}

\author{
Rui Hou \\ Guangdong University of Technology \\ School of Management
}

161 Yinglong Road, Tianhe District, Guangzhou, P. R. China, 510520

Phone: +86-20-8708-3656, +86-139-261-949-39

Email: hour@gdut.edu.cn

Jia-wen Wu

Guangdong University of Technology

School of Management

161 Yinglong Road, Tianhe District, Guangzhou, P. R. China, 510520

E-mail:wujwu@163.com

Helen S.Du

Guangdong University of Technology

School of Management

161 Yinglong Road, Tianhe District, Guangzhou, P. R. China, 510520

E-mail: hsdu@gdut.edu.cn

*Corresponding author: E-mail: hour@gdut.edu.cn

Address: 161 Yinglong Road, Tianhe District, Guangzhou, P. R. China, 510520

Phone \#: Phone: +86-20-8708-3656 Fax: +86-20-87083565 


\title{
Customer Social Network Affects Marketing Strategy: A Simulation Analysis Based on Competitive Diffusion Model
}

\begin{abstract}
To explain the competition phenomenon and results between QQ and MSN (China) in the Chinese instant messaging software market, this paper developed a new population competition model based on customer social network. The simulation results show that the firm whose product with greater network externality effect will gain more market share than its rival when the same marketing strategy is used. The firm with the advantage of time, derived from the initial scale effect will become more competitive than its rival when facing a group of common penguin customers within a social network, verifying the winner-take-all phenomenon in this case.
\end{abstract}

Keywords: customer social network; marketing strategy; competitive diffusion; population competitive model

\section{INTRODUCTION}

With the rapid development of information technology, history has witnessed the unprecedented importance of customer-to-customer interaction for marketing tools and strategies. Customers are inclined to shape their social network through various social media tools, in which they exchange ideas to get deeper recognition about targeted products. Also, such exchanges will undoubtedly have an impact on the potential customers who have the access to search for and read reviews or comments. Such virtual interactions based on the Internet makes it possible for customers with common interests to establish and maintain their social relationships with a more healthy and cost-effective method. Any small brand can gain its popularity by means of various network promotion strategies. That is to say, customer social networks, whether kinship-based or Internet-based, have become increasingly influential on theory research and practice of marketing tactics, proving to be of great importance to rethinking network marketing.

Over the years, there have been some significant changes in the factors of the consumer 
purchase decision. These factors start from independent purchase decisions, to the small-scale promotion by word-of-mouth amongst family and friends, and eventually developed into the large-scale internet-based targeted marketing (Jun et al.2006; Bao et al.2014)[1-2]. Nowadays, we are aware that the links amongst customers have become the base and premise of a trend setter (Provost 2009;Mochalova and Nanopoulos 2014)[3-4]. The interactions amongst customers plays an increasingly important role not only in maintaining brand image and enhancing customer loyalty (Zaglia 2013; Gamboa et al. 2014)[5-6], but also in affecting the formulation of marketing strategies for a certain brand or product (Yang et al. 2010)[7]. Such influence indicates that it is difficult for potential customers to make independent purchase decisions when they are influenced by the purchase behaviors of their surrounding friends (Robert et al. 2008)[8]. On the contrary, consumers are likely to be influenced by their surrounding friends and to take their choices as references when selective products or brands, which is also the basis of the network marketing theory (Tad 2010)[9]. With the rapid growth of Web2.0, the comments of customers will form an electronic word-of-mouth marketing effect, whilst opinion-leaders' comments has a profound effect on brand promotion (Brown et al. 2007; Momtaz et al. 2011)[10-11]. Individuals with certain positions or statuses within a social network have a larger influence than others because they are connected with more individuals within the social network. Thus, the key to success in virtual marketing or word-of-mouth promotion is the ability to identify and measure such influence (Kiss and Bichler 2008)[12]. Besides, network externality also has its role in influencing customer choices. For instance, for instant messaging software, individuals tend to choose the software their friends use. So we can assume that for each customer, his/her first choice must be the most popular amongst their friends. Apart from the loyal recommendation and the penguin effect, network externality matters in terms of potential customer purchase decisions and product promotion (Schoder, 1995; Thun et al., 2000)[13-14]. Once some customer chooses a certain kind of product, so will his friends and the friends of his friends. Such expansion forms the network-based viral marketing phenomenon (Yang et al. 2010; Jun et al. 2006; Zhu 2013)[7,1,15]. However, prior research is mostly limited to propagation of single information or product on a fixed network, and focuses on how the structure of customer social network influences the diffusion process, with the intent to formulate optimized marketing strategies (López-Pintado 2004; Aral and Walker 2011; Katona et 
al. 2011; Kim et al. 2011)[16-20].

Research on competitive propagation targeting no less than two kinds of information or product in one social network is limited. In recent years, competitive diffusion based on network has been concerned, such as the study of a minimum model of various epidemic fighting for host (Sneppen et al. 2010) [21] and a propagation model of cross-immune epidemic virus (Uekermann and Sneppen 2012)[22]. Like epidemic spreading processes, Granell et al. (2014)[23] discuss competitive spreading processes based on multilayered interconnected networks and information awareness interconnection. In terms of products competition and market expansion, by introducing a metaphor of biological systems, Kim et al.(2006)[19] built up a population competition diffusion model of two firms for competition equilibrium analysis and market share estimation, but they did not consider the links between consumers. By using population competition ideas, Yan and Ma (2011)[24] proposed a competition diffusion model in which two repeatedly purchased products compete in the same market, then discussed the conditions to achieve a competitive equilibrium state and the diffusion process; but did not consider the influence of customer social network. In the classic population competition equation, the preys are thought of as having no links with each other, independently scattered in a certain space. They are also regarded as passive, and can only wait to be hunted. However, a business ecosystem is very different. Here, customers are still thought as scarce resources to be hunted, but they have their own conscious and ability to make judgments instead of acting as a passive agent. In other words, we should consider the initiative of customers in social networks during the competition diffusion process.

Existing literature on spread of network primarily involve a single kind of information, product or epidemic on one complex network. But in daily life, consumers are more likely to be confronted with competitive promotion of more than two kinds of products, and they have more options when facing competitive propagation. In general, potential consumers will be undoubtedly influenced by their friend's decision when there is more than one option to choose from. So how exactly does customer decision preference influence the spreading process and result, if two or more products/brands diffuse on the same network competitively? To simplify the model, in this paper we assume that the consumers all have "penguin inclination", namely every individual is influenced by his/her friends, and are inclined to choose the product which is 
used by a larger proportion of his/her friends. With this assumption, we use QQ and MSN (China) as an example, to build up a population competition diffusion model based on consumer social network. And then we analyze the influence of network structure, initial conditions and marketing strategies on the competition diffusion process and the final equilibrium state by using simulation experiments. The potential contributions of this paper can be seen from two perspectives. Firstly, we propose a new population competition model based on customer social networks which can be used to explain how network structure affects the population competition process and the competition equilibrium state. Secondly, we find some optimal marketing strategies for two competitive products according to the simulation experiment results, which have significance in the guidance of the formulation of marketing plans in network marketing environment.

\section{WHY QQ WON MSN IN THE CHINESE MARKET}

In China's instant messaging market, the difference of market share between QQ and MSN (China) is not obvious in the early stages. However, several years later, as indigenous communication software, $\mathrm{QQ}$ is widely used. At the same time, as a foreign software, MSN (China)'s market growth is extremely slow. The final result is that QQ still dominates the market, despite MSN (China) efforts to catch up. And thus the following question arises, what made the large difference in market share between QQ and MSN (China)? With the same potential user groups, what is the determinant of the victory during the contest process between them?

In order to understand the competition process, we reproduce their growth history. QQ has gained rapid growth since its introduction in Feb, 1999. Up to Feb. in 2002, it was estimated that the number of QQ's registered users had risen to 20 million, with over one million active users. MSN was first put into service in USA in July, 1999 and had attracted seventy million users in the first six days. In May, 2005, it announced its official entry into China. In 2006, QQ accounted for $77.8 \%$ of the market share, whilst MSN (China) had $10.58 \%$. However, six years later, the market share of MSN (China) has shrunken to less than $5 \%$. The whole process is presented in Fig. 1, showing the active and registered user growth of QQ and MSN (China) individually. As time passes, the difference between the market shares of the two instant messaging companies becomes larger. All the data is obtained from Enfodesk. 

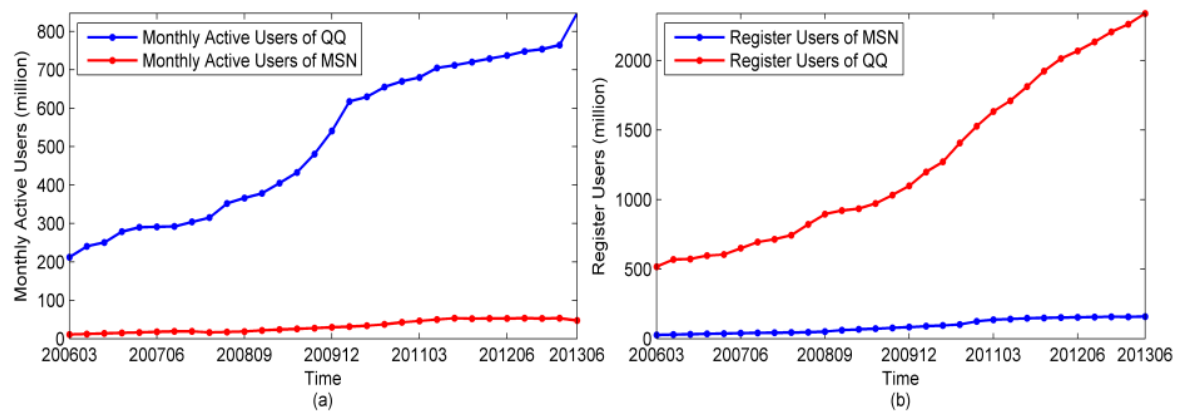

Fig. 1 Active and registered user growth of QQ and MSN (China)

Users and practitioners in the industry generally ascribe certain factors to MSN (China)'s shrinking market share. Technologically, MSN (China) bears its shortcomings in details, functions such as document delivery, screen interception, offline files, groups chatting cannot fulfill the expectations of consumers, whom are always troubled by the stability of the connection and non-delivery of messages. As for service, MSN (China) is usually criticized for the long waiting times for password recovery, slow feedback and poor service. Tactically, MSN (China) targets white-collars, which explains its failure in attracting the majority of netizens outside this target circle. The numbers of white-collars netizens, aggregated number of white-collar netizens and register users of MSN (China) are presented in Fig. 2. Users find it difficult to expand their social circles because MSN (China) users cannot add new friends without ID identification. All the Data is obtained from CNNIC, iReaserch and MSN (China).

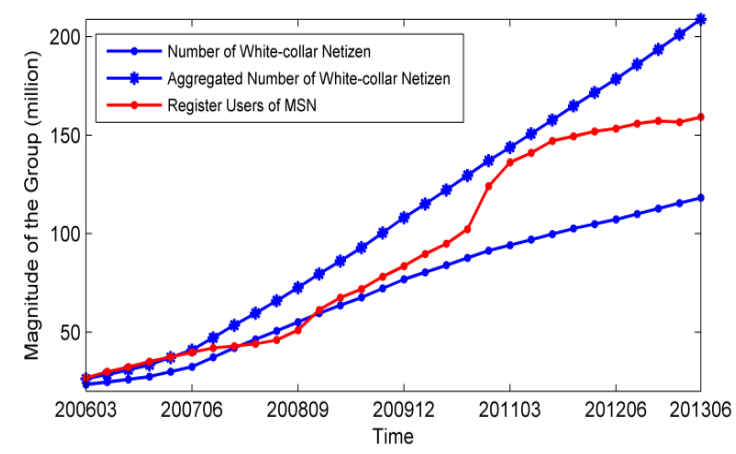

Fig. 2 User growth of MSN (China) from 2006 to 2013

However, the reasons mentioned above still fail to touch upon the root of this phenomenon. The reason for MSN (China)'s failure is that it ignores the inherent social networking features of China's netizens. MSN (China) should recognize that targeting white-collars as the primary target market is insufficient, because white-collars are just a portion of the potential users of instant 
messaging software. In other words, MSN (China) only captured a small part of the social network of China's netizens, the white-collar community network. MSN (China) did not provide sufficient means for the users in this community (white-collar) to connect users in other communities (e.g. blue-collar), which is the only way to expand the number of users rapidly. MSN (China) is inferior to $Q Q$ in two ways. Firstly, it's delayed entry to China's market caused it to lose the first-mover advantage. Secondly, it lacks the initial network scale effect, by which the network scale can be expanded rapidly based on simple rules, such as, potential users are inclined to choose software that is popular amongst their friends. However, friend relationship network is the key to success of instant messaging software. In this paper, we set up a new population competitive model based on social networks and verify these assumptions by simulation experiments.

\section{THE MODEL}

We assume that there are three groups in the customer network, group A for software product A's customers, group B for product B and group C for potential customers who do not use any similar products. Then, we can easily deduce that the contest between A and B not only lies in the potential market, but also the maximal conversion of the customers of the other product(s). In daily life, opinions of products are frequently exchanged within our social circles. People always have the desire to recommend products to those whom they are familiar with or to those whom they are associated with. More importantly, whether a potential customer would be follow a recommendation depends on how popular a preferred product is amongst the customer's friends. To be specific, the more friends that an individual has using product $A$, the more likely he/she will be persuaded into using product A, likewise for product B. If a recommendation is successful, it would then be classified to the addition of A or B correspondingly. In other words, competition is measured by the shift in the customers' scale of the two products.

Let $\rho_{1}(t)$ and $\rho_{2}(t)$ represent the customer scale of $\mathrm{A}$ and $\mathrm{B}$ separately, so the scale of potential customers can be calculated as $\left(1-\rho_{1}(t)-\rho_{2}(t)\right)$. Coefficients $\sigma_{1}\left(\sigma_{2}\right)$ are to show the competition constraints of one product upon the other. For instance, $\sigma_{1}$ refers to the limitation of product A's network externality which is caused by its increased customers against $\mathrm{B}^{\prime}$ s growth. This time the scale of potential customers for product $\mathrm{B}$ is $\left(1-\sigma_{1} \rho_{1}(t)-\rho_{2}(t)\right)$. Here 
network externality refers to the phenomenon that the influence of any one customer on others will be strengthened by the increasing customer scale of the product. When $\sigma_{1}>1$, the impact on B from A is large enough so that B's growth is bounded, resulting to the shrinking proportion of B's potential customers; When $\sigma_{1}=\sigma_{2}=1$, both A and B cannot restrict the scale of potential customers of the other, the network externality will be neutralized; If $\sigma_{1}<1$, we can assume that the contrary situation exists, which means that the impact on B from $A$ is small enough so that B's growth is unlimited, resulting in the expansion of B's potential customers, and some customers of A may shift to B.

In each round of competitive diffusion, there will be a loss of customers in both product A and product B. At the same time, the natural decay of customers will also affect the outcome of competition. Quite evidently, the quantity of growing customers can be calculated with the balance of the newly increased customers and the lost ones. Here, we define the decay ratio of the two products as $\delta_{1}$ and $\delta_{2}$. Therefore, the diffusion equations of the two products for the customers with the degree of $k$ (assuming the number of one's friends has amounted to $k$ ) during the competition process can be summarized as

$$
\begin{aligned}
& \frac{d \rho_{1 k}(t)}{d t}=-\rho_{1 k}(t) \delta_{1}+\left(1-\rho_{1}(t)-\sigma_{2} \rho_{2}(t)\right) g_{1, k} \\
& \frac{d \rho_{2 k}(t)}{d t}=-\rho_{2 k}(t) \delta_{2}+\left(1-\sigma_{1} \rho_{1}(t)-\rho_{2}(t)\right) g_{2, k}
\end{aligned}
$$

Here, $\rho_{1 k}(t)$ and $\rho_{1 k}(t)$ refers to the scale of group A and B with the degree of $k, g_{1, k}$ and $g_{2, k}$ refers to the possibility of the potential customers successfully recommended by group A and $\mathrm{B}$. As for potential customer $i$ whose degree is $k$, among his $k$ friends the number of friends in group $\mathrm{A}$ is $a_{i}$ and group $\mathrm{B}$ is $b_{i}$. Because of the network externality, the same recommendation process will be replicated again by every other customer after they are successfully recommended. Thus, competition diffusion phenomenon will emerge between product $\mathrm{A}$ and product $\mathrm{B}$.

We assume that at any particular round, customers can only make the recommendation once and the probability is the same for each successful recommendation. For a potential customer $i$, there would be several friends who use product A and B around him. Each of them 
has the possibility to recommend him a product according to their own product selection preference and thus the recommendation probability is up to its node degree, in other word, how many friends he/she has.
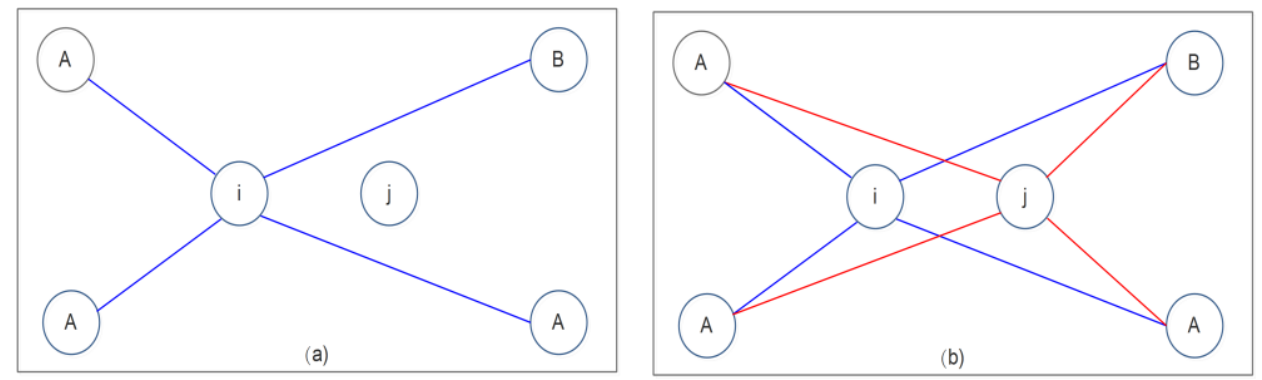

Fig.3 Impact of network structure on recommendation probability of success

As shown in formula (1) above, we can identify clearly that, for a potential customer, the probability of a successful recommendation is closely related to her/his social network structure. Since each individual can only recommend a friend once in each round, for potential customer $i$, the probability of a successful recommendation by customers of product A in fig. 3 (a) is twice as large as that in fig. 3 (b). This is because that another potential customer $j$ is added into the social network and will share the opportunity of being recommended by a customer of product A with potential customer $i$. This kind of recommendation mechanism shows that customer social network structure deeply affects the diffusion of product competition. Therefore, the calculation of the probability of a successful recommendation requires the introduction of network structure variable, node degree $k$.

In this paper, we use the mean field method, setting $\langle k\rangle$ as the average degree of customer social network, then $\frac{1}{<k\rangle}$ represents the probability that the potential customer $i$ can be recommended by a customer of product A or B.

However, whether potential customer $i$ uses product $\mathrm{A}$ or product $\mathrm{B}$, is up to the probability of a successful recommendation made by his/her friends. To simplify it, we assume that the probability is $r_{A}=r_{B}$. Therefore, the probability that potential customer $i$ is successfully recommended to adopt product $A$ will be

$$
p_{1, k, a, b}=r_{A}\left(1-\left(1-\frac{1}{<k>}\right)^{a}\right)
$$


Similarly, the probability that potential customer $i$ can be successfully persuaded to adopt product $B$ will be

$$
p_{2, k, a, b}=r_{B}\left(1-\left(1-\frac{1}{<k>}\right)^{b}\right)
$$

Obviously, for a group of potential customers with $k$ degree, the friends around him will be a collection of all possible combinations of customers of $A$ and $B$. By considering all the possible combinations of the number of A and B's customers as well as the probability of a successful recommendation for each combination, we will get the function of the probability that potential customers with $k$ degree can be recommended to use product A:

$$
g_{1, k}=\sum_{a=0}^{k} \sum_{b=0}^{k-a} p_{1, k, a, b}\left(\begin{array}{l}
k \\
a
\end{array}\right)\left(\begin{array}{l}
k-a \\
b
\end{array}\right) \theta_{1}(t)^{a} \theta_{2}(t)^{b}\left(1-\theta_{1}(t)-\theta_{2}(t)\right)^{k-a-b}
$$

Here, $\theta_{1}(t)$ and $\theta_{2}(t)$ refers to the link probability between the potential customer and his friend who are using product $\mathrm{A}$ and $\mathrm{B}$ separately, where $\theta_{1}(t)=\frac{1}{<k>} \sum_{k \geq 1} k P(k) \rho_{1 k}(t)$ and $\left.\theta_{2}(t)=\frac{1}{<k>} \sum_{k \geq 1} k P(k) \rho_{2 k}(t) \cdot \rho_{1 k}(t) 、 \rho_{2 k}(t)\right)$ refers to the ratio of the quantity of product A and B's customers with $k$ degree and the quantity of all the customers with $k$ degree in the network separately.

Similarly, the probability that potential customers with $k$ degree be recommended to use product $B$ is

$$
g_{2, k}=\sum_{a=0}^{k} \sum_{b=0}^{k-a} p_{2, k, a, b}\left(\begin{array}{l}
k \\
a
\end{array}\right)\left(\begin{array}{l}
k-a \\
b
\end{array}\right) \theta_{1}(t)^{a} \theta_{2}(t)^{b}\left(1-\theta_{1}(t)-\theta_{2}(t)\right)^{k-a-b}
$$

After combining the functions $(1) \sim(5)$, we can get the population competitive diffusion equations of these two products based on customers social network:

$$
\begin{aligned}
& \frac{d \rho_{1 k}(t)}{d t}=-\rho_{1 k}(t) \delta_{1}+\left(1-\rho_{1}(t)-\sigma_{2} \rho_{2}(t)\right) g_{1, k} \\
& \frac{d \rho_{2 k}(t)}{d t}=-\rho_{2 k}(t) \delta_{2}+\left(1-\sigma_{1} \rho_{1}(t)-\rho_{2}(t)\right) g_{2, k} \\
& g_{1, k}=\sum_{a=0}^{k} \sum_{b=0}^{k-a} r_{A}\left(1-\left(1-\frac{1}{<k>}\right)^{a}\right)\left(\begin{array}{l}
k \\
a
\end{array}\right)\left(\begin{array}{l}
k-a \\
b
\end{array}\right) \theta_{1}(t)^{a} \theta_{2}(t)^{b}\left(1-\theta_{1}(t)-\theta_{2}(t)\right)^{k-a-b} \\
& g_{2, k}=\sum_{a=0}^{k} \sum_{b=0}^{k-a} r_{B}\left(1-\left(1-\frac{1}{<k>}\right)^{b}\right)\left(\begin{array}{l}
k \\
a
\end{array}\right)\left(\begin{array}{l}
k-a \\
b
\end{array}\right) \theta_{1}(t)^{a} \theta_{2}(t)^{b}\left(1-\theta_{1}(t)-\theta_{2}(t)\right)^{k-a-b}
\end{aligned}
$$




\section{SIMULATION}

\subsection{Simulation purpose}

For the population competition diffusion equation, we assume the maximum node degree of the customer social network is $\mathrm{M}$, so the amount of equations is $2 \mathrm{M}$. It is less likely for us to figure out the analytic equilibrium solutions. In this case, with a premise of customer penguin inclination (that is, customers tend to choose the most popular product amongst his/her friends) and a fixed social network, we observe the impact that customer initial parameters and marketing strategies makes on the competition process and the ultimate equilibrium results by using a simulation experiment. We hope the results can be useful for developing a firm's marketing strategy.

\subsection{Simulation ideas}

For competitive products like QQ and MSN (China), we think it is necessary to simulate their competition process as well as market growth and that it is worthwhile to find and explore the practical strategies for population competition. Despite the failure to change or control the structure of potential customer social network, after probing into the network's structure and making use of the possible spreading effect of seed customers, we can still accelerate the acceptance and expansion of one product among customers, and finally reach the goal of rapidly enlarging the market scale and winning the opponents. In other words, how can a firm choose the target seed customers within an established customer social network?

In this paper, we are about to draft two different strategies for seed customer selection. We first set the following parameters: $S_{i}$ is for the initial scale of seed customers, $p(k)$ for the probability that product $i$ choice for seed customers with degree of $k$, and $s_{i}(k)$ for the selection proportion.

STRATEGY 1: Product $i$ chooses seed customers randomly, in which case we fix the same proportion for different node degrees. So STRATEGY 1 can be functional as:

$$
\left\{\begin{array}{l}
s_{i}(k)=S_{i} \\
\sum_{k=1}^{M} s_{i}(k) p(k)=S_{i}
\end{array}\right.
$$


STRATEGY 2: Product $i$ would preferably select those with the highest node degree as seed customers. To put it in practice, we should first select the potential customers with highest node degree as seed customers until the scale of them reach the standard limit, $S_{\lim i t}$. Then we choose those with the next largest node degree as seed customers, and so on, until the whole scale of seed customers reach $S_{i}$. So STRATEGY 2 can be functional as:

$$
\left\{\begin{array}{l}
s_{i}(k)=S_{\text {lim } i t}, k>l \\
s_{i}(k)=S_{i}-\sum_{k=l+1}^{M} s_{i}(k) p(k), k=l \\
\sum_{k=l}^{M} s_{i}(k) p(k)=S_{i}
\end{array}\right.
$$

Here, $l$ refers to the smallest node degree of seed customers that can assure its smallest scale $S_{i}$.

For the two competitive products, there are two factors of growth--the loss of present customers and the external network effect on potential customer choices. Here, external network effect refers to the phenomenon that potential customer preference for one product will be intensified with the product's customer scales. As the overall demand scale is fixed, the increasing number of customers of one product will cause the customers of the other product to decrease. We name such mutual suppression as competitive inhibition coefficient, $\sigma_{1}\left(\sigma_{2}\right)$.

In the real world, for some social networks it is hard to say that they are a scale-free network because of their small size, but on-line social networks are different as their sizes are very big, such as QQ and MSN (China) community networks referenced in this paper. Therefore, we set our simulations on scale-free networks. As for premises, we assume the maximum node degree of customer social network as $M=150$, the average node degree of the network as $<k>=10$ and that node degree distribution obeys the following rule:

$$
P_{S F}(k)= \begin{cases}\beta k^{-\alpha} & k \leq M \\ 0 & k>M\end{cases}
$$

In this paper, the MATLAB mathematics software is used in simulation experiments, and the simulation program and algorithm are as follows. 
STEP 1: Generate the scale-free power-law distribution $P_{S F}(k)$. Customer social network is the carrier of the competition diffusion process, therefore, in this paper we need to generate a scale-free power-law distribution $P_{S F}(k)$ to represent the connection characteristics of customer communities. According to the degree distribution law in formula (9), we must find parameters $\alpha$ and $\beta$ to meet $\sum_{k=1}^{M} k \cdot \beta k^{-\alpha}=<k>$. Because the sum of proportions of all kinds of nodes degree is 1 , namely $\sum_{k=1}^{M} \beta k^{-\alpha}=1$, then $\frac{\sum_{k=1}^{M} k \cdot k^{-\alpha}}{\sum_{k=1}^{M} k^{-\alpha}}=<k>$, we can calculate the approximate value of parameter $\alpha$, thus we get the degree distribution of $P_{S F}(k)$.

STEP 2: Generate the degree distributions of seed customers both product $A$ and $B$ in the initial state of simulation. Base on this customer social network, whether product A or product B, is required to decide the distribution of seed customers. According to the competitive strategy, we then generate the degree distributions of seed customers with various node degree one by one, both product A and B. For example, in the initial state, the market share of seed customers with degree $k$ of product $\mathrm{A}$ is $\rho_{1}(k)=s_{1}(k)$, while the market share of seed customers with degree $k$ of product B is $\rho_{2}(k)=s_{2}(k)$.

STEP 3: Update the degree distributions of seed customers both product $A$ and $B$ in the net simulation step. According to the competitive diffusion mode, namely formula (6), we can update the degree distributions of seed customers both A and B, $\rho_{1}(k)$ and $\rho_{2}(k)$ in every round.

STEP 4: Adjust control variables and output simulation results. According to the different simulation scenarios and objectives from part 4.3.1 to 4.3 .4 , we then adjust the control variables, $\delta, \sigma$, STYATEGY 1 or 2 , and market entering timing, to get all kinds of simulation results in competitive diffusion process between product $A$ and product $B$. 


\subsection{Simulation results}

\subsubsection{Considering customer's natural decay and network externality effect}

This simulation aims to analyze how the competitive inhibition from network externality impacts on the final equilibrium of the two products. The simulation parameters are set as follows: (1) The same natural decay ratio, $\delta_{1}=\delta_{2}=0.01$; (2) the same initial seed customers scale, $S_{1}=S_{2}=0.0001$. (3) and the same strategy, STRATEGY 1 as their guidance, that is, they both choose seed customers randomly.

With that the only variant of the simulation experiment is the different competition inhibition coefficients, $\sigma_{1}\left(\sigma_{2}\right)$. We begin our experiment based on the rules of population competition diffusion showed as equation (6), and the simulation result is showed in fig.4.
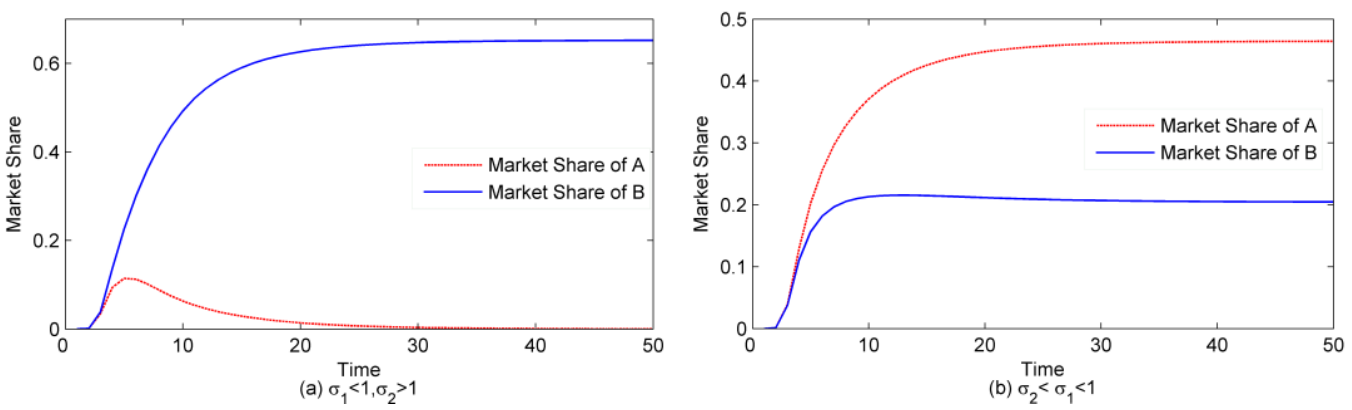

Fig.4. Impact of competition inhibitions coefficients on diffusion process and equilibrium state

The chart above shows that the product with stronger network externality effect is on its way to a larger market share because of its larger restraint on its rival, if the same strategy is adopted for both products. The larger the competition inhibition coefficient is, the smaller the number of potential customers is left for the opponents, and finally the greater the capacity for its growth. In addition, when one's inhibition coefficient is greater than 1 whilst the other is less than 1 , the former is capable of winning established customers from the weaker (latter) side, which accelerates the shrinking market share of the weaker to finally reach the dominant position in the market. That is the "Winner Take All" (WTA) phenomenon. Otherwise, if inhibition coefficient of both are less than 1, they are all free to fight against each other for more customers, in which case the weaker side would not be forced to quit and both sides can co-exist in the market. 


\subsubsection{Not considering customer's natural decay and network externality}

As the structure of customer social network cannot be changed in short time, pragmatic value would be added if we fix the social net structure and observe the impact on competition process and equilibrium state caused by different marketing strategies. At the very start of the simulation, to exclude interference from different natural decay conditions and competitive inhibition coefficient, we let $\delta_{1}=\delta_{2}=0, \sigma_{1}=\sigma_{2}=1$. Then all our attention will be paid on the impact caused by strategy choices and the scale of seed customers.

(1) Impact of different strategies with the same seed customer scale

Strategy here refers to the two strategy patterns mentioned above. In reality, it is less likely for us to select all the potential customers with degree of $k$ as seed customers because of budget restraints. So we set proportion limits for seed customers, $S_{\lim i t}=0.1$ (In the following simulation, the limits are the same), and the same initial customers scale for both sides, $S_{1}=S_{2}=0.0001$. Assuming that product A applies STRATEGY 2 and B applies STRATEGY 1, we get the result as showed by fig. 5 .

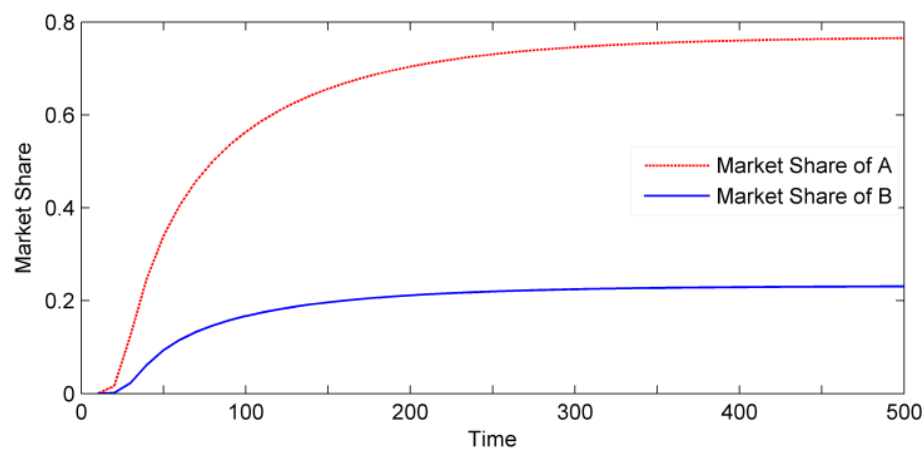

Fig.5 Impact on competition diffusion process and equilibrium from different marketing strategy

The chart shows seed customer selection strategy has distinct influence on the process. When competitive equilibrium is reached, product A with STRATEGY 2 gains a larger market share than product B with STRATEGY 1. With shared premium of seed customers, STRATEGY 2 chooses the customers with larger node degrees as seed customers in the social network, which allows product $A$ to have an increased probability of successful recommendations. Besides, the absence of natural decay also maintains product A's superiority and finally makes product A the absolute winner in the market. 
(2) Impact of the same strategy with different seed customer scales

To figure out the difference made by initial scale of seed customers, we begin another simulation with a base on shared customers social network and strategy. We assume $S_{1}=0.0001 、 S_{2}=0.0005$ and both products apply STRATEGY2. The result is showed in Fig.6.

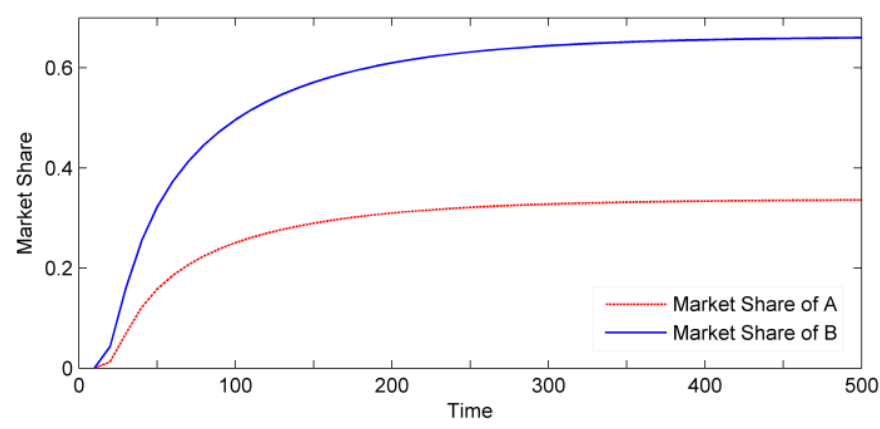

Fig.6 Impact from seed customers scale on competition diffusion process and equilibrium state

Seen from the chart, product B with seed customer scale advantage would finally beat product $A$ in terms of market share when competitive equilibrium state is reached. The larger the initial scale of seed customers, the stronger the influence on potential groups in the early stage of competition diffusion, and the greater the amount of market share it will obtain at equilibrium. Therefore, when customer social network is fixed and the two products choose the same marketing strategy, the initial scale of seed customers has an important influence on the competition diffusion process and the equilibrium state.

\subsubsection{Strategy advantage V.S. first-mover advantage}

There is another situation we should consider: assuming product B gets into the market earlier (the leader) but adopts STRATEGY 1 for random selection of seed customers, while product $A$ enters the market later (the follower) with STRATEGY 2. To begin the observation, we first set the same initial scale $S_{1}=S_{2}=0.0001$. That is, we assume that product A is equipped with strategy advantage and product B with the first-mover advantage. Our question is what is the best timing for product $\mathrm{A}$ to get into the market if it wants to beat product B? Fig.7 is the simulation result. 


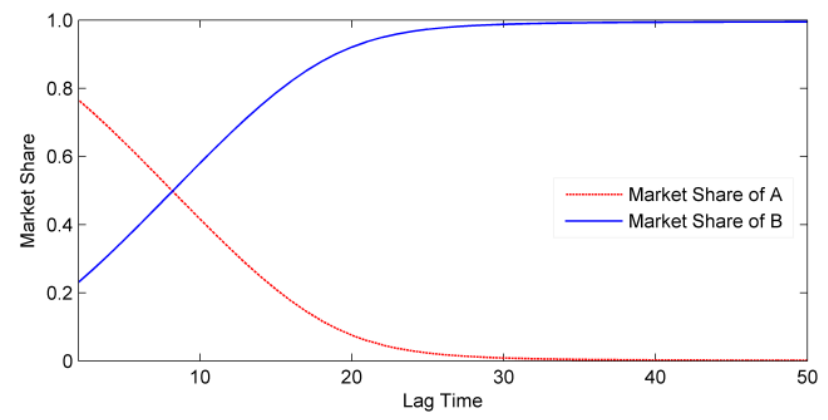

Fig.7 The relationship between lag time and market share of two products

Here, lag time means the length of time between when product $A$ and product $B$ enter the market. For example, QQ entered the China instant messaging software market in Feb. 1999 whilst MSN (China) in May 2005, so the lag time is 75 months. The simulation result shows that with the help of strategic advantage it is still possible for the follower to catch up to the leader provided that the lag time is short enough. However, the strategy advantage cannot always make up for the lag time. Besides, the longer the follower is left behind, the smaller market share it will have. Hence, there will be an optimal timing for the follower to enter the market so that it can catch up with the leader.

\subsection{Response to the case}

The results we conclude from the simulation help explain the competition between $Q Q$ and MSN (China) as we described in the case. Firstly, despite their respective network externality and their ability to take customers from each other, QQ still has a superior market share against MSN (China) due to its larger initial scale of customers which limits MSN (China)'s growth. They co-exist in the Chinese market but there is a large difference between them in terms of market share. Secondly, even if the network externality effect is excluded, faced with QQ's large market share, MSN (China) still can't win with its delayed entry into China's market. As a result, MSN (China) is incapable of catching up with QQ in spite of its advantageous technology and marketing strategy. In reality, because of the fixed social network structure of Chinese netizens and tendency for the penguin effect, QQ's first-mover advantage allows it to dominate its victory in the Chinese market. 


\section{CONCLUSIONS}

This paper has built up a population competition diffusion model based on the customer social network. In this model, we assume no difference between the two products and that the probability for the potential customer preference depends on the choice of the individuals' surrounding friends. For competitive firms, although they cannot change the established customer social network structure, they can still have room for growth by making full use of the characteristics and the laws of such a structure, to gain a larger market share by strategically selecting seed customers who have important roles in the network, as well as larger seed customer scale. As a result, in order to research the impact on the competition process and the equilibrium state from the perspective of customer social network structure and selection strategy, we have build up a stimulation experiment and reached the following conclusion.

Firstly, with a shared marketing strategy and decay rate for customers, the product with a stronger network externality effect, that is, with a bigger competitive inhibition coefficient, will ultimately grasp a larger market share. In addition, if either one of the two products has a competitive inhibition coefficient greater than 1, the "winner take all" (WTA) phenomenon occurs. Only when both products have a competitive inhibition coefficient smaller than 1 , can they co-exist in one competitive market, where the product with a smaller competitive inhibition coefficient will get a smaller market share.

Secondly, when excluding the impact of customer natural decay and network externality, a firm can gain competitive advantage through superior selection strategy of seed customers, (larger) initial seed customer scale and (earlier) timing for market entry. To be specific, the advantage is larger if the initial seed customers selected have higher node degree (namely, with more friends) rather than choosing randomly. That is to say, a firm with strategic advantage can gain a larger market share than a rival if the other conditions are the same. In addition, the larger the scale of initial seed customers a firm has, the more competitive advantage it can get, if the other conditions are the same.

Thirdly, by ruling out the influence from customer natural decay and network externality, the first product entering the market will have initial scale advantage compared to the following product. With a similar entry time into the market, the product with delayed entry can still catch 
up or even surpass the pioneer by making use of strategic advantages. However, if the lag time is long enough, there is nothing the product with delayed entry can do.

Our paper shows that under the customer social network environment, with little difference between products, penguin customers and the same marketing strategy, the firm with timing advantage derived from the initial scale effect will be more competitive than its rival, and have the advantage to some extent, to hamper on the opponent's growth, which is a powerful explanation for the WTA phenomenon.

\section{ACKNOWLEDGMENTS}

This research is supported by National Science Foundation of China (No.71571052, 71572050).

\section{REFERENCES}

[1] Tackseung Jun, Jeong-Yoo Kim, Beom Jun Kim, M.Y. Choi. Consumer referral in a small world network. Social Networks 28 (2006) 232-246

[2] Tong Bao, Tung-lung Steven Chang. Finding disseminators via electronic word of mouth message for effective marketing communications. Decision Support Systems 67 (2014) 21-29

[3] Provost F, Dalessandro B, Hook R, et al. Audience selection for on-line brand advertising: privacy-friendly social network targeting[C]//Proceedings of the 15th ACM SIGKDD international conference on Knowledge discovery and data mining. ACM, 2009: 707-716.

[4] Anastasia Mochalova, Alexandros Nanopoulos. A targeted approach to viral marketing. Electronic Commerce Research and Applications 13 (2014) 283-294

[5] Melanie E. Zaglia. Brand communities embedded in social networks. Journal of Business Research 66 (2013) 216-223

[6] Ana Margarida Gamboa, Helena Martins Goncalves. Customer loyalty through social networks: Lessons from Zara on Facebook. Business Horizon 57(2014) 709-717

[7] Jianmei Yang, Canzhong Yao, Weicheng Ma, Guanrong Chen. A study of the spreading scheme for viral marketing based on a complex network model. Physica A 389 (2010) $859-870$

[8] East Robert, Kathy Hammond, Wendy Lomax. Measuring the Impact of Positive and Negative Word of Mouth on Brand Purchase Probability. International Journal of Research in 
Marketing 25 (2008) 215-24.

[9] Hogg Tad. Inferring preference correlations from social networks. Electronic Commerce Research \& Applications 9 (2010) 29-37

[10] Brown J, Broderick A J, Lee N. Word of mouth communication within online communities: conceptualizing the online social network. Journal of interactive marketing 21 (2007) 2-20

[11] Niyoosha Jafari Momtaz, Abdollah Aghaie, Somayeh Alizadeh. Identifying opinion leaders for marketing by analyzing online social networks. International Journal of Virtual Communities and Social Networking 3 (2011) 43-59

[12] Christine Kiss, Martin Bichler. Identification of influencers - measuring influence in customer networks. Decision Support Systems 46 (2008) 233-253

[13] Schoder, D. Diffusion yon Netzeffektgütern. Marketing Zeitschrifi für Forschung und Praxis(Germany), 17 (1995) 18-28.

[14] Jörn-Henrik Thun, Andreas Größler. Peter M. Milling. The diffusion of goods considering network externalities: a system dynamics-based approach. 18th International Conference of the System Dynamics Society, Bergen, Norway, 8 August 2000.

[15] Zhiguo Zhu. Discovering the influential customers oriented to viral marketing based on online social networks Physica A 392 (2013) 3459-3469

[16] Dunia López-Pintado. Diffusion in complex social networks. Games and Economic Behavior $62(2008) 573-590$

[17] Aral S, Walker D. Creating social contagion through viral product design: a randomized trial of peer influence in networks. Management Science 57 (2011) 1623-1639

[18] Katona Z, Zubcsek P P, Sarvary M. Network effects and personal influences: the diffusion of an online social network. Journal of Marketing Research 48 (2011) 425-443

[19] Jonghwa Kim, Deok-Joo Lee, Jaekyoung Ahn. A dynamic competition analysis on the Korean mobile phone market using competitive diffusion model. Computers \& Industrial Engineering 51 (2006) 174-182

[20] Kim S, Lee K, Cho J K, et al. Agent-based diffusion model for an automobile market with fuzzy TOPSIS-based product adoption process. Expert Systems with Applications 38 (2011) 7270-7276.

[21] Kim Sneppen, Ala Trusina, Mogens H Jensen, Stefan Bornholdt. A minimal model for 
multiple epidemics and immunity spreading. PloS ONE 5 (2010) 1-7

[22] Uekermann F, Sneppen K. Spreading of multiple epidemics with cross immunization. Physical Review E 86 (2012) 036108

[23] Clara Granell, Sergio Gómez, Alex Arenas. Competing spreading processes on multiplex networks: awareness and epidemics. Physical Review E 90 (2014)

[24] Yan H S, Ma K P. Competitive diffusion process of repurchased products in knowledgeable manufacturing. European Journal of Operational Research 208 (2011) 243-252 